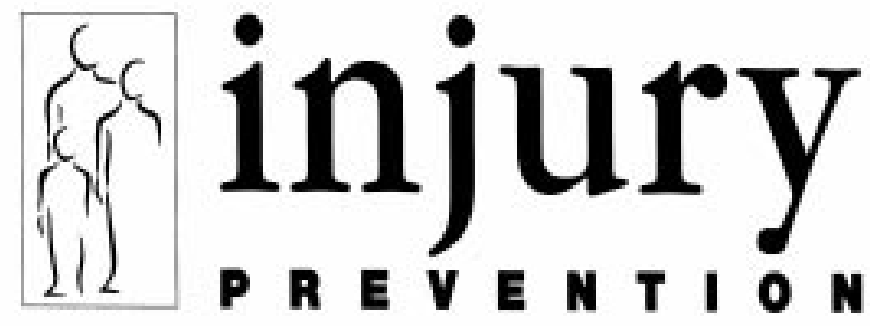

Journal of the International Society for Child and Adolescent Injury Prevention

\title{
Editorials
}

\section{Alcohol: the ubiquitous risk factor}

This issue includes four papers that draw attention to one of the most important causes of injury in all age groups and in every part of the world-alcohol. In particular, the papers address impaired drivers. The first is an original article pointing to the benefits of laws that penalize drivers whose blood alcohol content (BAC) exceeds $0.08 \%$ by weight ( $p$ 109). In view of the growing evidence to which this paper adds substantially, it is difficult to understand why so many constituencies tolerate higher limits. It is even more difficult to fathom why, whatever the limits, more impaired drivers are not apprehended or, when they are, why they are not punished more severely.

These issues are addressed in the second paper-one that we hope will be the first of many written by from a lawyer's perspective ( $p$ 96). The senior author, Robert Solomon, is the legal advisor to the Canadian branch of Mothers Against Drunk Driving (MADD). Although the statistics in this paper only reflect the experience in one province, my guess is that the issues these data point to are commonplace, not only elsewhere in Canada but also world wide. In spite of limitations in the data system itself, the findings clearly point to serious flaws in how both the police and the legal system address impaired driving. Set aside the legal subtleties and look at the larger picture the findings present. As someone who has been spared personal experience with drunk driving, I find the results extremely disturbing. Imagine, then, how the families of victims must feel about this "system" that is supposed to deter.

Because most injury prevention initiatives depend on lay persons for their success, I asked the vice president of MADD in the US, Wendy Hamilton, to present it as a Featured Program ( $p$ 90). Anyone familiar with this amazing program is bound to be impressed with its many accomplishments. Sadly, its origins and continued strength derive almost entirely from needless personal tragedies. There are many such programs in other countries and each deserves similar recognition.

Finally, because impaired driving is dealt with so differently from one country to another, I asked Kathryn Stewart of the Pacific Institute for Research and Evaluation to write a guest editorial on this issue ( $p$ 80). She graciously agreed. Her superb contribution draws attention to how international the effort has been to conquer this problem. Not surprisingly, perhaps, the country with the lowest tolerated level is Sweden, $0.02 \%$. Stewart's editorial also addresses varying sanctions or penalties for violations, and in particular, the contentious issue of the role of previous offenders. As well, she examines licensing policies and experiences with enforcement, especially the use of sobriety checkpoints.

Before I elaborate further on the issue of alcohol as a risk factor, I feel obliged to disclose my interest. Last year, after publishing the essay by Marielle Olivier about the killing of her sister by a drunk driver, I asked her to keep me informed about the events that followed. I was appalled by her bewilderingly discouraging account of the court case, and how powerless she and the other members of the family felt. Consequently, I became involved with MADD Canada and was recently appointed to its National Board of Directors.

Injury Prevention readers need no reminding that alcohol is not just a risk factor for road crashes. The risk in almost every other category of injury-unintentional and intentional-especially house fires, drowning, sports, and recreation, is also greatly increased by the presence of alcohol. Thus, alcohol is a pervasive risk factor, frequently compounded, especially in the case of road crashes, by drug use (illicit, prescribed, or over-the-counter).

It is evident that eliminating or sharply reducing the use of alcohol in risk situations is the most direct route to success. What is much less evident is how best to achieve this goal. Is stronger legislation more effective? Does it really matter how vigorously laws are enforced? Should these strategies remain foremost, or should more emphasis be placed on technological solutions such as driver interlock devices? My instincts tell me that of the alternatives available, better legislation is still the most certain path to success. However, as Solomon points out, legislation that may appear "strong" may be much less so if other aspects of the law make it difficult to enforce. In Ontario, and undoubtedly in many other constituencies, policing and judicial problems remain unresolved. This is also true for helmet laws, seat restraint use, smoke detectors, and life jacket use.

As in so many other injury prevention issues, it seems that the goal will be reached more quickly and efficiently when health departments play a larger part. Health must join with justice, transport, and many other branches of government, to overcome the obstacles that remain. Whether these involve the reluctance of police to do random breath tests or that of judges to hand out stiff sentences, one underlying explanation is almost certain to include the 
widespread acceptability of alcohol in most societies. In part this reflects how alcohol is portrayed on TV, in magazines, etc. Nowadays when we see an old movie or video in which it seems everyone is smoking, we are surprised and perhaps disapproving. But we react differently when we see alcohol being consumed or when beer ads are shown during high profile events, especially sporting events.

The evolution of tobacco control measures is instructive. Focusing on the place of alcohol in society, may, therefore, be the next step. The day may not be too far off when governments will launch class action suits against alcohol manufacturers to recover some of the cost of the care of those injured by this product just as they have done with tobacco. We need to reconsider the logic of allowing the sale of alcohol to remain relatively unrestricted. Without wishing to appear as if I were advocating a return to the days of prohibition, we cannot afford to view this product much differently than we now do its fellow evil spirit, tobacco.
Would it help if products containing alcohol were better labeled to reflect their dangers, as many countries now do for cigarettes? Whatever the answer, it is evident that conventional preventive measures such as strict enforcement of impaired driving and minimum legal drinking age laws, sobriety checkpoints, and prompt suspension of licenses have brought us only part of the way towards the goal. According to a recent Morbidity and Mortality Weekly Report from the Centers for Disease Control and Prevention in the US, the percentage of alcohol related traffic fatalities in the US remained at about 38\% between 1997 and $1998 .{ }^{1}$ With only the measures now in place, the Surgeon General's target cannot possibly be met in the foreseeable future.

One way or another, we must act more effectively against alcohol used in excess. It is unlikely that there is any other risk factor that accounts for so many preventable injuries.

1 Anonymous. MMWR Morb Mortal Wkly Rep 1999;38:1086-7.

\section{Still more on helmets: setting an example}

Even if I am rarely successful, at least I am persistent. After my recent radical decision to publish my own letter to the editor of the $B M \mathcal{F}$ on the subject of helmet laws, I have been embroiled in a similar controversy in Quebec. Our transport minister is considering such legislation, but the antihelmet lobby is trotting out all the usual chestnuts. This lobby usually consists of pro-bicycling groups. Perhaps as a consequence of having not worn helmets for too long, they are convinced that a helmet law is anticycling. I regard it as quite the opposite. In any event, after an essay by the president of Le Monde a Bicyclette appeared in the Montreal Gazette, I wrote a rebuttal using the same arguments as I gave in my letter to the BMF. The Gazette was hesitant to publish my contribution because the editor thought it sounded too much as if only doctors knew what was best when it came to head injuries. I pleaded guilty.

I also recently wrote an editorial for the Canadian Medical Association fournal, commenting on a paper examining trends in spinal cord injuries in amateur hockey in Canada. Helmet use (and misuse) is also an issue in this sport, and the juxtaposition of bicycling with hockey reminded me of an article in my favorite journal-the Medical Post. It was about a legendary ophthalmologist, Dr Tom Pashby, shown in these marvelous photos. The article described this 84 year old surgeon's long campaign to require hockey players to wear helmets.

Forty years ago, his 14 year old son was concussed when his head hit the ice during a hockey game. At the time, the neurosurgeon bluntly told Pashby he was "stupid" to let his son play hockey without a helmet. Pashby then began to lobby for a "no helmet-no hockey" rule. He also insisted that all minor league hockey players be required to wear helmets with visors or face guards for eye protection.

The payoff for amateurs is impressive. Over the last 23 hockey seasons there were 1860 eye injuries and 298 blinded eyes. In 291 of the latter, the players were not wearing a visor. In comparison, last year there were only five serious eye injuries in amateur hockey in Canada.

For better or worse, professional athletes are often seen as role models, by adults as well as children. What these millionaires wear, and how they behave, has a profound influence on much of the public, as do the regulations under which their sport operates. Although professional hockey players are now obliged to wear helmets, it appears that the regulation neglects the obvious requirement that the chinstrap be properly fastened.

Professional players who choose not to wear a visor face a different picture than amateurs who do. In mid-February, for the second time in one week, two players on the Montreal Canadiens hockey team were hit in the face by a puck. One, who had a previous eye injury, still declines to wear a visor. "No, I've been playing this way too long to change now", was his thoughtful answer. The other had his larynx crushed by a puck. Ironically he had become a visor wearer after a serious eye injury. Recently a third player lost an eye when struck by a puck. He will never play again. Another escaped blindness by millimeters but will miss several games because of blood in the eye. Dr John Little, the Canadiens ophthalmologist, has been advising players to wear visors for over 20 years.

Undoubtedly, some players believe there is a stigma attached to visors. They are often abused and taunted by fellow players for choosing to do so. One TV commentator argues that "feisty" players hide behind their visors; that it is a sign of weakness; that it gives them a false sense of security, and leads to more high sticks. The latter is unproven, but even if it were true, the league need only enforce existing
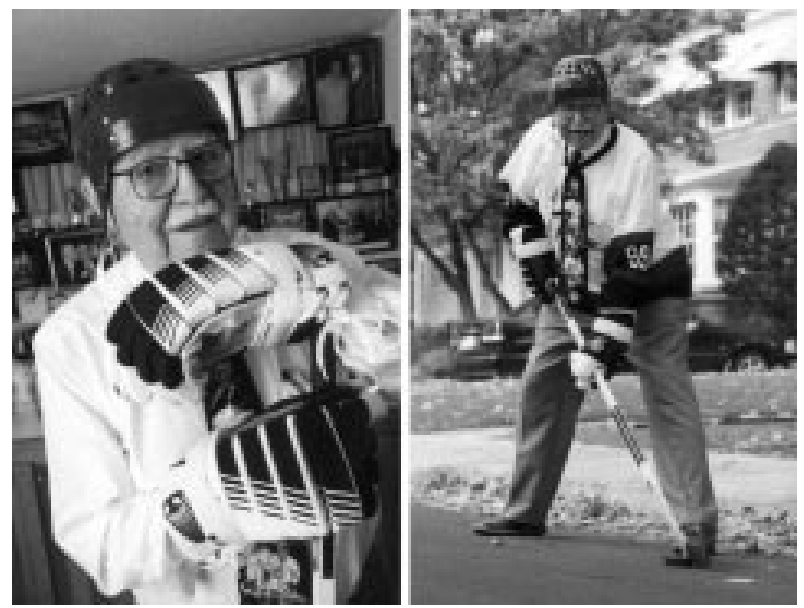

Thanks to Stephane Ulraney for permitting us to use these photos. 
regulations to remedy this. It is agreed that visor use will only happen when the league insists, just as it has compelled players to wear helmets. The fact that the league does not require visors is irresponsible and sets a bad example for younger players. As does the fact that well known players have been pictured wearing helmets with the straps dangling loose.

These examples makes one wonder how much those responsible for this, or other professional sports, care about the safety of their players, let alone the example such behavior sets for others, especially the young. As Dr Pashby realized, sports injuries at all levels are frequent and often very serious. Many are preventable by the proper use of proper gear but many others can only be prevented by strong regulations governing players' behaviour. It goes without saying that such regulations are useless if they are not consistently enforced.

\section{The review process}

Academics know the limitations of evaluations that fall short of what is required to infer causality. If an evaluation fails to eliminate or minimize biases, the program has a weak claim to success. Put simply, "bias" in this context refers to other explanations for attributing a success (or failure) to what is being evaluated than the program itself. Few evaluations are able to overcome all such biases and most good reviewers alertly spot them.

Tough reviews are never ignored. When sharply critical comments are received, it is likely a paper will be rejected. If it is not, it is often because another reviewer was less censorious. In such cases, authors are invited to submit a revision-with no promises of acceptance-and the decision is deferred. Occasionally, more than one revision is required. Final decisions are made by the editor alone, and although scientific merit is the most important consid- eration, it is not paramount. We strive to achieve a balance of topics, countries and disciplines, and to include papers that resonate with frontline workers.

In this issue, three papers fall into this admittedly less than perfect category. None employ the best possible design for an evaluation. They appear, however, because on balance, it seemed that each presented approaches and other elements that could prove useful for others in similar situations. Although we have no intention of encouraging second rate research of any kind, it is important to acknowledge that good evaluation research is usually costly, time consuming, and may require expertise that is often difficult to find. To comment further and provide some guidance on how to balance these issues, Sue Gallagher has written an accompanying editorial.

\section{More advice to contributors}

In the last issue, I reminded contributors of Original Articles that we had a 3000 word limit. I do so again and now broaden this contributor advisory to include the cardinal rule for any author-always read the instructions to authors in the journal of your choice. Then check that your paper meets all the requirements. Neglecting this all important rule will at least delay the review process. At worst, it may have a negative influence on the judgment of the editor or reviewers. (Of course, the best way to ensure that your paper fits is to be familiar with the journal-that is, to subscribe.)

A common offense is failing to use approved Index Medicus abbreviations of journal titles in the references. But where are these to be found? In the past, I used to beg, borrow, or steal, from our hospital library, old copies of the List of Serials Indexed for Online Users issued by the US Department of Health and Human Services. Nowadays, it is possible to download all abbreviations from a web site, but this is extremely time consuming. Not being sure how best to help contributors find these abbreviations, I placed the question on several list servers. Almost immediately I received over 20 replies. About one half wondered why any journal was still insisting on these abbreviations and urged that we abandon the rule. Until we do so, the simplest solution for those with access to the web is to go to the Pubmed site. The full address is http://www.ncbi.nlm.nih.gov/PubMed, but usually just Pubmed will do. Enter the full title of the journal, et voila, the proper official abbreviation!
Next, a word about keywords: these are important and should be chosen with care. They need not include "injury" or "prevention" because the indexers, given the journal title, automatically include both terms. What else appears is up to you; MESH terms are always preferable but these often fail to serve the injury prevention field well. The best way to decide on what is needed is to put yourself in the position of someone searching for your paper. Which search terms would you use, apart from those in the title, if you wanted to find your paper quickly? Once your paper is published, because Injury Prevention is listed in Index Medicus and other databases (not all journals meet the rigid requirements for such inclusion), a useful lesson is to search for your paper, and when you find it, see what terms the indexers have added.

We now invite submissions by e-mail (preferably in Word, Wordperfect, or RTF). This saves time, money, trees, and permits rapid feedback if there are omissions or problems that need to be resolved before the paper is sent for review, often by e-mail.

One final new request: in future, all contributing authors will be asked to submit one or more lists of bulleted key points. These will be "boxed" and placed in suitable positions in the text to help the reader find the main messages more easily. (Another goal of this announcement is to see if anyone reads these editorials!)

I B PLESS Editor 


\section{Program evaluation — balancing rigor with reality}

Funders require it. Practitioners fear it. Epidemiologists narrowly focus it on outcomes. Perspectives on evaluation vary, but there is general agreement that we need more of it in the injury field. The question is how much? and what kinds of evaluation can be done in a real world situation, with limits on resources? Randomized control trials may be the "gold standard" and quasi-experimental design a close second choice, but most of us do not have the luxury of engaging in such studies. Rigorous and multilevel evaluations that encompass formative (design), process (implementation), and outcome (knowledge, behaviors, injury rates, and institutionalization) measures are still relatively rare in the injury prevention literature.

Four articles in this issue (see pages 125, 130, 151, and 154) illustrate the range of possibilities as well as many of the difficulties inherent in evaluating community based programs. Three of the four employ multiple strategies in a pre-post design, with varying outcome measures. Although none of these studies is perfect, there is, nevertheless, much that can be learned from them.

The study of Bhide et al (p 154) contains some elements of a process evaluation by examining the effectiveness of the manner of distribution of a one shot, prepackaged educational program. It presents the number exposed to the program and the components that were implemented as intended, for example the leader's guide. Given the minimal penetration (16\%) and only partial implementation $(46 \%)$, it is unlikely that a better designed outcome based evaluation would have shown more encouraging, statistically significant changes. In fact, if the researchers had only used an outcome evaluation, we may have concluded that the program was not effective, when, in fact, the real issues are the penetration and its incomplete implementation. This study clearly shows why an essential part of evaluation is determining whether a program has been implemented as planned. And, in this case, it illustrates the special difficulties when programs must depend on schools to distribute the prevention material.

In the study of Lee et al (p 151), one element of an outcome evaluation of a three year bicycle helmet promotion campaign is presented. The study uses a comparison of self reported helmet wearing among teenagers in intervention and control sites as proxy measures for the injuries of interest. Despite the somewhat greater, but modest, increase in self reported helmet wearing over the five year period, many questions are left unanswered. Process measures, describing the extent of implementation, or the inclusion of observed helmet use as an outcome measure (as noted by the authors), would provide greater assurance that the conclusions are valid. They would also assist others who wish to replicate this approach. The use of a control group is an advantage of this study, but adds to the complexity. This is evident in the inability to obtain accident and emergency reports of injuries as planned. It also raises questions about possible contamination of the control sites by television and radio coverage of the campaign.

In contrast, the study of Hanfling et al (p 125) uses actual observations of the proper use of seat belts and car seats to evaluate the short term effects of a six component public education campaign. The study compares two intervention sites to control sites after nine months of intervention. Such a design has a higher level of methodological rigor than the previous examples and lends credibility to the positive results. The use of control groups is clearly an asset, but does not answer all questions. As in the previous study, the use of a multilevel intervention approach with several partners probably contributed to the positive findings, but it is next to impossible to conceive of methods that would determine the contribution of each component, that is, was one element of the intervention more effective than another. This study also identified potential confounding variables that spring up in assignment of control sites. It illustrates the complications that so often arise when trying to obtain good controls. Finally, had they included process measures, such as exposure to the interventions, the number of incentives, or number of citations issued by the police, this might have explained why the rate of restraint use achieved was much less than that reported in countries with well enforced legislation.

The study of Coggan et al (p 130) is the most comprehensive, ambitious, and rigorous in its evaluation. It is based on a multitargeted community injury prevention program that combines both qualitative and quantitative data. The investigators include a multilevel process and outcome evaluation, with intervention and control sites matched on several variables. They conducted a formative evaluation to choose the interventions for each of the targeted audiences. The qualitative data enhance our understanding of implementation details and illuminate the successful aspects of the program. The case studies provide the information needed to adapt programs to the culture. It is clearly the most sophisticated but probably the most costly of the four studies. Knowing more about the penetration rate might influence the replicability of this program, but these details are not given.

So where does this leave us? Despite the best intentions, constraints on evaluation are inevitable for community programs. What do we need to do a better job?

\section{Achieving balance}

Regardless of the size of the program and its budget, some level of evaluation can and always should be performed. We should not be debating whether quantitative or qualitative research is better. Formative, process, and outcome measures are inter-related and are meant to complement each other. It is not a question of either/or, but rather a question of finding the best combination to adequately address the goals of the evaluation. Program staff and evaluators will do a better job if they work together right from the beginning to set clear, detailed goals and objectives for intervention programs and their evaluation. This approach is bound to achieve better evaluation priorities.

\section{Training}

The extent and nature of the evaluation is often determined by the expertise of the researcher or the program manager. The tools of epidemiology are critical for improving the evaluation of community based programs but are enhanced when combined with those from other disciplines. There is much more to evaluation than outcome measures, such as morbidity and mortality. Although high level evaluation methods may not always be feasible or may be too costly, a greater barrier to their use is often the lack of training. The need to expand research training opportunities was one of the key recommendations in the recent report by the Institute of Medicine. Ide- 
ally, researchers should be skilled in understanding the constraints of doing an evaluation in real community settings.

\section{Increasing resources}

Multilevel evaluation research is more expensive and often requires a longer follow up period than traditional grant awards of three years or less. Funders need to be persuaded to change their award structure to accommodate truly comprehensive, rigorous program evaluations. Without their support, or a creative linkage of several funders, we will not be able to improve the quality of evaluation research.

The state of the art of evaluation, especially of community programs, has not evolved as much as we would like in the last two decades. The logistics and difficulties in managing one intervention in one community, let alone a complex set of interventions in several communities simultaneously, and evaluating their efficacy, continues to be a great challenge. In spite of this, there is much to learn from the kinds of studies published in this issue of the journal. They help us to better understand the critical elements of a successful or unsuccessful intervention, and highlight both a program's true worth and its limitations. Nevertheless, we can do better and must strive to do so.

SUSAN S GALLAGHER

Center for Injury and Violence Prevention,

Education Development Center, 55 Chapel Street,

Newton, MA 02458-1060, USA

e-mail: sgallagher@edc.org 